\title{
Survival of Spoilage and Pathogenic Microorganisms on Cardboard and Plastic Packaging Materials
}

\author{
Lorenzo Siroli ${ }^{1}$, Francesca Patrignani, ${ }^{1,2}$, Diana I. Serrazanetti ${ }^{2}$, Cristiana Chiavari ${ }^{3}$, \\ Marzia Benevelli ${ }^{3}$, Luigi Grazia ${ }^{3}$ and Rosalba Lanciotti1,2*
}

\begin{abstract}
${ }^{1}$ Department of Agricultural and Food Sciences, Alma Mater Studiorum, University of Bologna, Campus of Food Science, Cesena, Italy, ${ }^{2}$ Interdepartmental Center for Industrial Agri-Food Research, Alma Mater Studiorum, University of Bologna, Cesena, Italy, ${ }^{3}$ Department of Agricultural and Food Sciences, Alma Mater Studiorum, University of Bologna, Reggio Emilia, Italy
\end{abstract}

The aim of this work was to study the interaction of corrugated and plastic materials with pathogenic and spoiling microorganisms frequently associated to fresh produce. The effect of the two packaging materials on the survival during the storage of microorganisms belonging to the species Escherichia coli, Listeria monocytogenes, Salmonella enteritidis, Saccharomyces cerevisiae, Lactobacillus plantarum, Pseudomonas fluorescens, and Aspergillus flavus was studied through traditional plate counting and scanning electron microscopy (SEM). The results obtained showed that cardboard materials, if correctly stored, reduced the potential of packaging to cross-contaminate food due to a faster viability loss by spoilage and pathogenic

Edited by:

Paula Teixeira,

Universidade Católica Portuguesa,

Portugal

Reviewed by:

Giuseppe Spano,

University of Foggia, Italy

Fohad Mabood Husain,

King Saud University, Saudi Arabia

*Correspondence:

Rosalba Lanciotti

rosalba.lanciotti@unibo.it

Specialty section:

This article was submitted to

Food Microbiology,

a section of the journal

Frontiers in Microbiology

Received: 25 September 2017 Accepted: 14 December 2017 Published: 22 December 2017

Citation:

Siroli L, Patrignani F, Serrazanetti DI,

Chiavari C, Benevelli M, Grazia L and Lanciotti R (2017) Survival

of Spoilage and Pathogenic Microorganisms on Cardboard and Plastic Packaging Materials.

Front. Microbiol. 8:2606

doi: 10.3389/fmicb.2017.02606 microorganisms compared to the plastic ones. In fact, the cell loads of the pathogenic species considered decreased over time independently on the inoculation level and packaging material used. However, the superficial viability losses were significantly faster in cardboard compared to plastic materials. The same behavior was observed for the spoilage microorganisms considered. The SEM microphotographs indicate that the reduction of superficial contamination on cardboard surfaces was due to the entrapping of the microbial cells within the fibers and the pores of this material. In addition, SEM data showed that the entrapped cells were subjected to more or less rapid lyses, depending on the species, due to the absence of water and nutrients, with the exception of molds. The latter spoilers were able to proliferate inside the cardboard fibers only when the absorption of water was not prevented during the storage. In conclusion, the findings of this work showed the reduction of cross-contamination potential of corrugated compared to plastic packaging materials used in fruit and vegetable sector. However, the findings outlined the importance of hygiene and low humidity during cardboard storage to prevent the mold growth on packaging.

Keywords: entrapping, pathogens, safety, cardboard, plastic, scanning electron microscopy

\section{INTRODUCTION}

In the last few years, various food-borne illnesses have been attributed to the consumption of fresh vegetables and fruits. Norovirus, Salmonella spp., Listeria monocytogenes, and Escherichia coli O157:H7 are reported to be the leading cause of fresh produces related outbreaks (Alegre et al., 2010; Scallan et al., 2011; Oliveira et al., 2012; Siroli et al., 2014; Callejón et al., 2015). Pathogenic 
and spoilage microorganisms can contaminate fresh products in different phases of the product processing and, given the absence of treatments that can eliminate microorganisms, can reach the end consumer (Francis et al., 2012). Moreover, the maintaining of refrigerated temperatures throughout the whole supply chain is often very difficult and thermal abuses can lead to an increase of microbial growth rates both on the packaging surfaces and the products (Kusumaningrum et al., 2003). In addition, the long-lasting permanence of some microorganisms on the package surface of fresh produces can lead to their rapid multiplication and possible biofilm formation (Barnes et al., 1999; Uhlich et al., 2006; Wilks et al., 2006; Shi and Zhu, 2009). The presence of biofilm can provide protection to pathogenic and spoilage microorganisms that can survive longer and be involved in cross-contamination phenomena from packaging to food (Bae et al., 2012; Valeriano et al., 2012). The packaging of fresh products, and in particular its microbiological quality, can play a very important role both in the quality and safety of the product. Very limited and fragmentary information regarding the microbial cell loads present on the surfaces of packaging materials are available in literature. Nevertheless, the presence of pathogens and spoilage microorganisms on the packaging surfaces is documented (Suominen et al., 1997; Mafu et al., 2011; Ismaill et al., 2013). Differences in chemical/physical characteristics, correct storage, and sanitizing of packing materials strongly affect the microbial loads present on the packaging surfaces. In fact, reusable packaging generally presents aerobic mesophilic loads between 3 and $6 \mathrm{log} \mathrm{CFU} / \mathrm{cm}^{2}$, while virgin fiber packaging presents cell loads ranging between 2 and $5 \log \mathrm{CFU} / \mathrm{cm}^{2}$. Several microorganisms were detected on the packaging surfaces and spore forming bacteria such as Bacillus spp. and Clostridium spp. and molds such as Aspergillus spp. and Cladosporium spp. are the more involved (Suominen et al., 1997; Binderup et al., 2002; Turtoi and Nicolau, 2007; Patrignani et al., 2016). Nevertheless, the presence of pathogenic microorganisms in not well-sanitized packaging material is reported in literature (Patrignani et al., 2016). Given this situation it is important to select an appropriate packaging based on the product characteristics and to prevent possible microbial contamination (Campos et al., 2014). Corrugated cardboard packaging is one of the most widely used types for packaging fresh products and presents significant advantages from an environmental and microbiological standpoint than plastic materials (Levi et al., 2011; Hladikova et al., 2015).

As things stand at present, it is extremely complicated to determine how and under what role the packaging can contribute to microbial cross-contamination of the packaged product. In fact, the microbial presence on packaging surfaces as well as on packed fruits and vegetables is difficult to establish because it has a very high variability (Siroli et al., 2014; Patrignani et al., 2016). Additionally, the storage conditions such as temperature and relative humidity of the packed products, and the eventual presence of biofilms and availability of nutrients play a key role in the survival and multiplication of microbial communities, affecting the possibility of cross-contamination from the package to the fresh product (Siroli et al., 2014; De Candia et al., 2015). However, cross-contamination phenomena from packaging to the product are also influenced by the characteristics of the packed fruit or vegetable as the degree of ripeness, acidity, sugar content, and presence of physical damages (Heaton and Jones, 2008).

Recently Patrignani et al. (2016) demonstrated, trough challenge tests and a modeling approach, a higher transferring of the microorganisms E. coli, Pseudomonas spp., and Saccharomyces cerevisiae in peaches packed in reusable plastic containers (RPC) compared to corrugated cardboard. The authors demonstrated a reduction of potential cross-conta mination from packaging to fruits in cardboard compared to plastic as well a higher microbiological quality of peaches stored in cardboard boxes. These authors hypothesized that the better microbial qualities and the reduction of crosscontamination of cardboard compared to RPC were due to the capability of cardboard to entrap microbial cells. Also Brandwein et al. (2016) studied the relationship between corrugated cardboard packaging and fresh produce and the potential of an anti-biofilm polymer to coat corrugated cardboard surfaces in order to mitigate surface bacterial biofilm formation. However, to our knowledge, the literature data on this topic are still scarce. Thus, this research was aimed to study the interaction of corrugated and plastic materials with different microbial species, such as pathogens (L. monocytogenes, E. coli, Salmonella enteritidis) and spoiling (S. cerevisiae, Lactobacillus plantarum, Pseudomonas fluorescens, Aspergillus flavus) ones. In particular, the effect of the two packaging materials on the survival during the storage of the target microorganisms was studied through traditional plate counting and scanning electron microscopy (SEM). To reach the research goals, the target microorganisms were inoculated, at the same levels, on corrugated and plastic surfaces and their microbial cell loads were monitored over time. In addition, to understand the fate of the inoculated microorganisms, the packaging material sections were studied by SEM.

\section{MATERIALS AND METHODS}

\section{Microbial Strains}

The microbial strains employed in this work were E. coli 555, L. monocytogenes SCOTT A, S. enteritidis E5, S. cerevisiae spa, Lb. plantarum 82, P. fluorescens 4T04, and A. flavus belonging to the Department of Agricultural and Food Sciences of Bologna University.

Escherichia coli 555, L. monocytogenes SCOTT A, S. enteritidis E5, and $P$. fluorescens 4T04 were preliminarily grown on Brain Heart Infusion (BHI) Broth (Oxoid Ltd., Basingstoke, United Kingdom) and incubated at $37^{\circ} \mathrm{C}$ for 24 h. S. cerevisiae and $A$. flavus were grown in Yeast Extract Peptone Dextrose (YPD) broth (Oxoid Ltd., Basingstoke, United Kingdom) at $25^{\circ} \mathrm{C}$ for $48 \mathrm{~h}$ and $L b$. plantarum 82 was grown on De Man, Rogosa, and Sharpe (MRS) broth (Oxoid Ltd., Basingstoke, United Kingdom) at $37^{\circ} \mathrm{C}$ for $24 \mathrm{~h}$. 

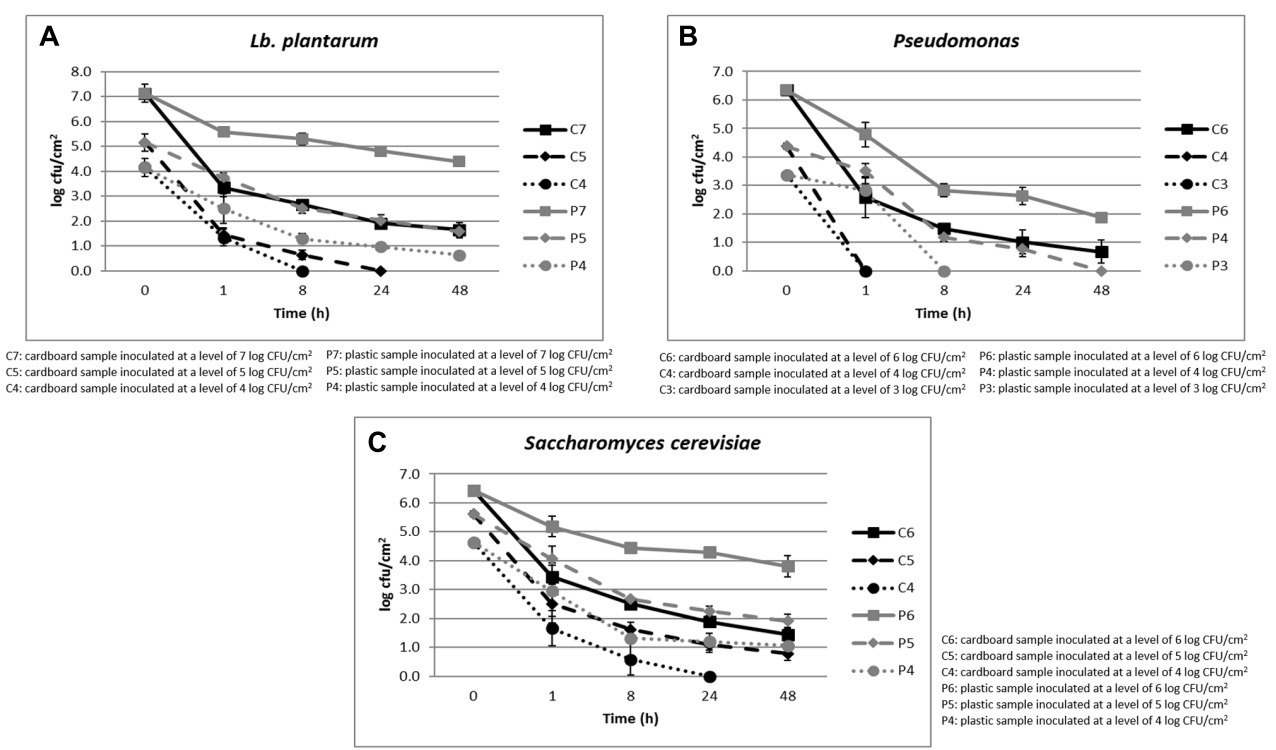

6: cardboard sample inoculated at a level of $6 \log \mathrm{CFU}_{\mathrm{cm}}^{2}$

C5: cardboard sample inoculated at a level of $5 \mathrm{log} \mathrm{CFU} / \mathrm{cm}^{2}$
C4: cardboard sample inoculated at a level of $4 \log \mathrm{CFW} / \mathrm{cm}^{2}$

P6: plastic sample inoculated at a level of $6 \log \mathrm{log} / / \mathrm{cm}^{2}$ PS: plastic sample inoculated at a level of $5 \mathrm{log} \mathrm{CFU} / \mathrm{cm}^{2}$
P4; plastic sample inoculated at a level of $4 \mathrm{log} \mathrm{CFU} / \mathrm{cm}^{2}$

FIGURE 1 | (A-C) Cell loads recorded during the storage at environmental temperature of $L$ b. plantarum (A), P. fluorescens (B), and S. cerevisiae (C) in relation to the inoculation levels and the packaging materials.
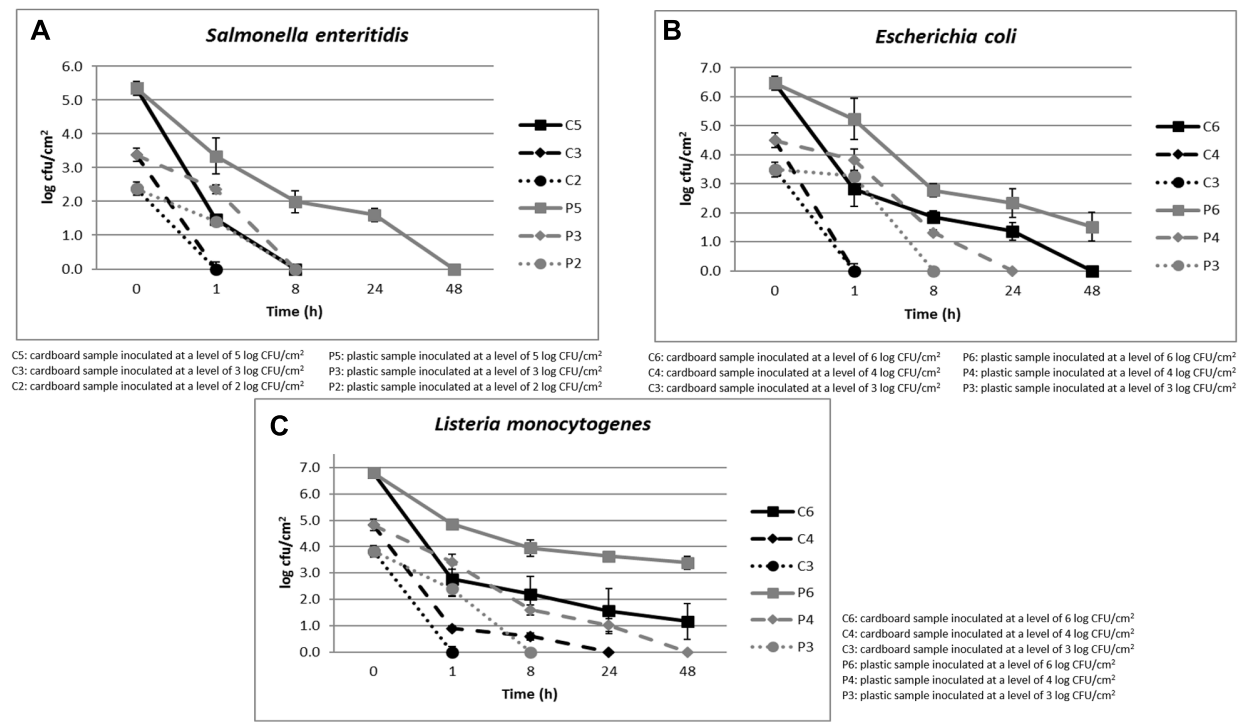

C6: cardboard sample inoculated at a level of $6 \log \mathrm{CF} / \mathrm{cm}^{2}$
C4: cardboard sample inoculated at a level of $4 \log \mathrm{CFU} / \mathrm{cm} \mathrm{m}^{2}$

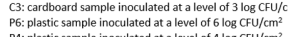

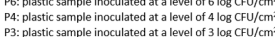

FIGURE 2 | (A-C) Cell loads recorded during the storage at environmental temperature of S. enteritidis (A), E. coli (B), and L. monocytogenes (C) in relation to the inoculation levels and the packaging materials.

\section{Entrapping Capability of Corrugated Compared to Plastic}

Corrugated and plastic surfaces were used for studying the entrapping capability of each material. The size of the surfaces was $5 \mathrm{~cm}^{2}$ both for corrugated and plastic.

The target microorganisms, pre-cultivated as reported in the section "Microbial Strains," and refreshed overnight in the appropriate growth media were inoculated in the considered surfaces separately and at three different inoculum levels (ranging between 2.0 and $6.0 \log \mathrm{CFU} / \mathrm{cm}^{2}$ ). The microorganisms were prepared by making serial dilutions in sterile physiological solution $(0.9 \% \mathrm{NaCl})$. The $5 \mathrm{~cm}^{2}$ surfaces were inoculated with $500 \mu \mathrm{l}$ of the appropriate dilution. The inoculated surfaces were dried at room temperature $\left(25^{\circ} \mathrm{C}\right)$ for $30 \mathrm{~min}$ and sampled after $1,8,24$, and $48 \mathrm{~h}$. To recover the microorganisms, a swabbing method (Ismaïl et al., 2013) was adopted by using a classic hygienic cotton swab moistened in sterilized peptone water $0.1 \%$ (Oxoid Ltd., Basingstoke, United Kingdom). Then 

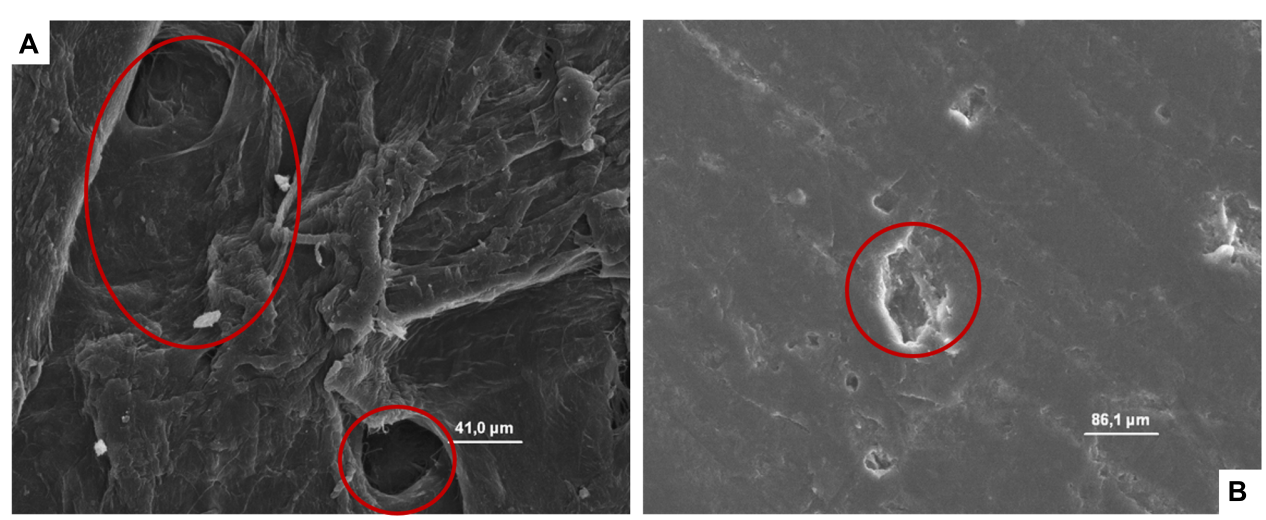

FIGURE 3 | (A, B) SEM microphotographs of cardboard (A) and plastic (B). In A the presence of pores is evident, while in B a smooth, plane, and homogeneous surface with the presence of a clear scratch is evident.
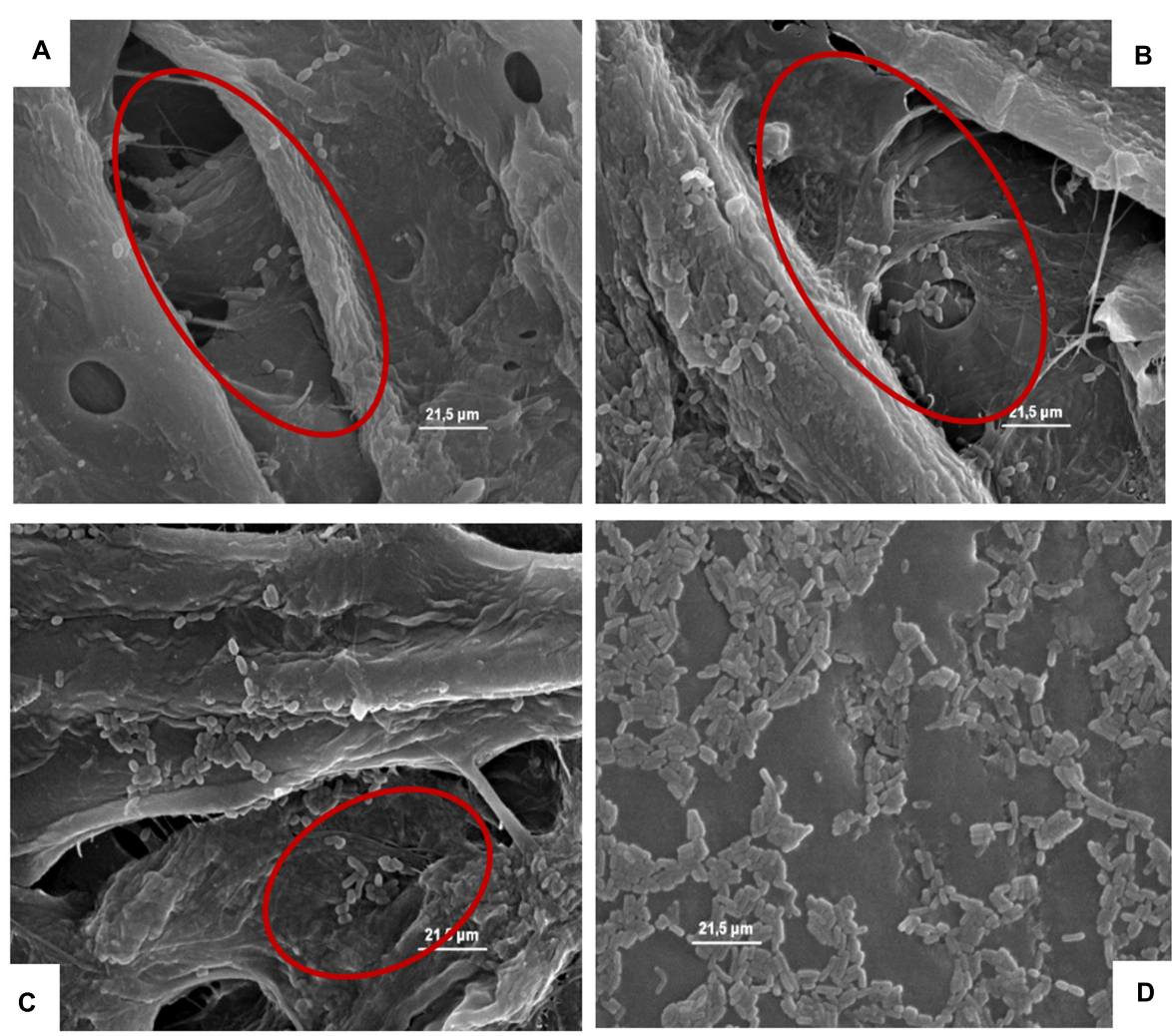

FIGURE 4 | (A-D) Presence of the inoculated $L b$. plantarum on cardboard surface after $1 \mathrm{~h}$ (A), $24 \mathrm{~h}$ (B), $48 \mathrm{~h}$ (C), and after $1 \mathrm{~h}$ on plastic surface (D). In A-C the presence of microbial cells in pores formed between cardboard fibers is evidenced. In $\mathbf{D}$, it is highlighted that all the microbial cells remain all on the surface of the packaging material.

the swab was immersed in $10 \mathrm{ml}$ of maximum recovery diluent (MRD) (Oxoid Ltd., Basingstoke, United Kingdom) and if necessary serial dilution in MRD broth was performed. Finally, the microorganisms were enumerated in selective media. In particular, Listeria Selective Agar Base (Oxoid Ltd., Basingstoke, United Kingdom) was used for the enumeration of Listeria, Violet Red Bile Agar (Oxoid Ltd., Basingstoke, United Kingdom) for E. coli, Bismuth Sulfite Agar (Oxoid Ltd., Basingstoke, United Kingdom) for the detection of Salmonella, Pseudomonas Agar Base (Oxoid Ltd., Basingstoke, United Kingdom) for Pseudomonas, YPD for the enumeration of S. cerevisiae, and MRS (Oxoid Ltd., Basingstoke, United Kingdom) for the enumeration of $L b$. plantarum. For each analysis, three independent repetitions were performed in different days. 

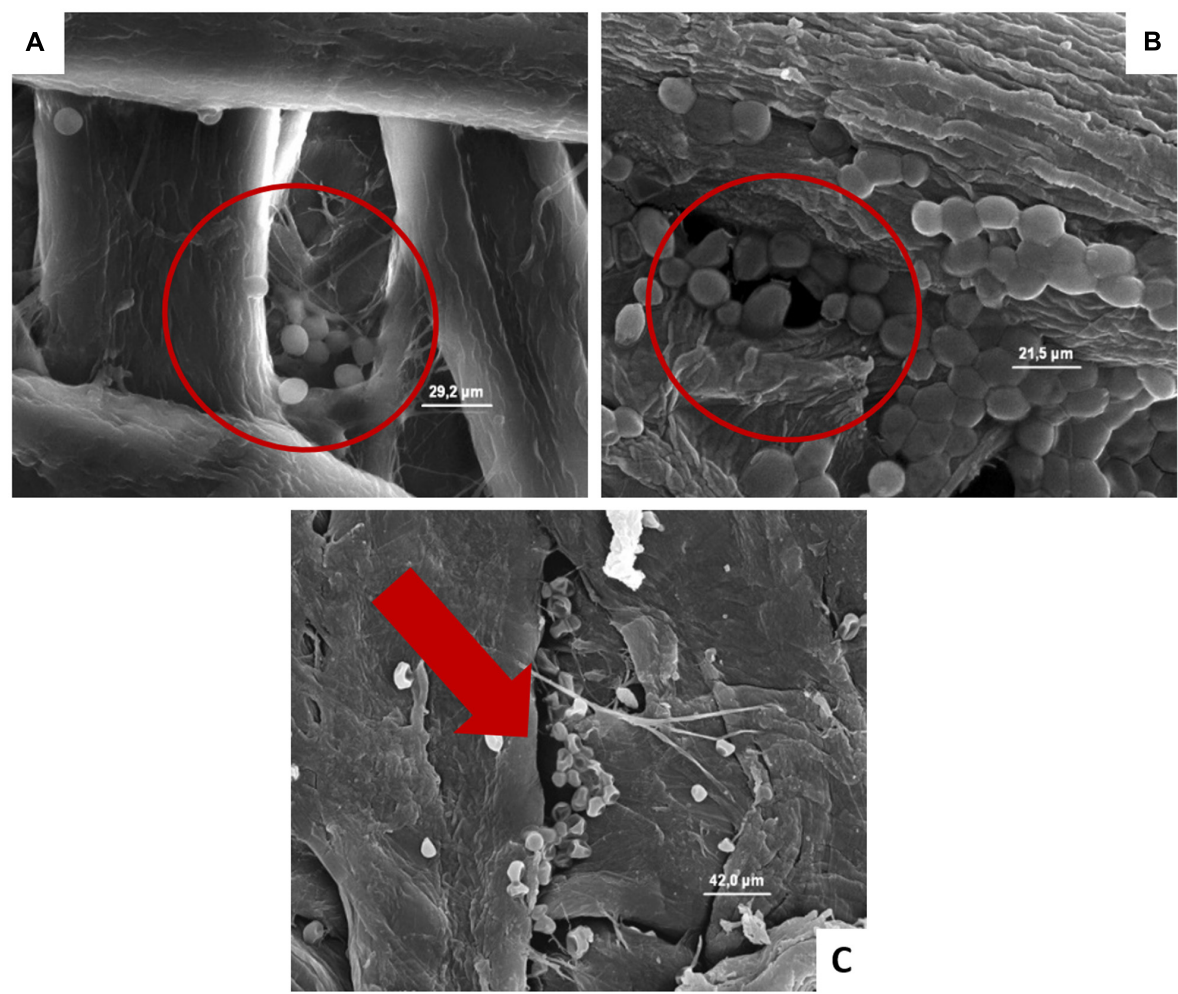

FIGURE 5 | (A-C) Presence of the inoculated S. cerevisiae on cardboard surface after $1 \mathrm{~h}$ (A), $24 \mathrm{~h}$ (B), and $48 \mathrm{~h}$ (C). In $\mathbf{A}$ and $\mathbf{B}$ is evidenced the entrapping of microbial cells in pores formed between cardboard fibers. In $\mathbf{C}$ the cell lyses also of entrapped cells is outlined by the red harrow.

\section{Scanning Electron Microscopy (SEM) Analyses}

The SEM analyses were performed on corrugated surfaces, inoculated separately with at a level of $6 \mathrm{log} \mathrm{CFU} / \mathrm{cm}^{2}$ with L. monocytogenes, E. coli, S. cerevisiae and Lb. plantarum, and A. flavus after 1, 24, and $48 \mathrm{~h}$. Moreover, a trial on corrugated surfaces inoculated with a mixture of all the target microorganisms was performed. Furthermore, analyses on plastic surfaces not inoculated and inoculated with $\mathrm{Lb}$. plantarum were performed.

Relative to bacteria and yeasts, the method suggested by Sowden and Walker (1988) was applied, introducing modifications concerning the preparation of individual samples.

After the inoculations small pieces of packaging were cut out with a razor blade and dehydrated stepwise in ethanol (50-100\%) at room temperature after critical point the specimens were mounted on SEM discs, coated with golds, and observed using a Hitachi S-510 SEM (Hitachi, Tokyo, Japan).

For A. flavus observation, due to the mold fragility, the protocol suggested by Edelmann and Klomparens (1994) was employed. The samples were firstly fixed with glutaraldehyde and then dehydrated with the graded ethanol series (50-100\%).

\section{Statistical Analysis}

Microbiological data were examined with STATISTICA software v. 8.0 (TIBCO Statistica, Palo Alto, CA, United States) and a two way-ANOVA followed by Fisher's least significant difference (LSD) test at $p<0.05$ were performed.

\section{RESULTS AND DISCUSSION}

\section{Cell Loads of Spoilage Microorganisms in Relation to Inoculation Level, Packaging Material, and Storage Time}

The cell loads recorded during the storage at environmental temperature of $L b$. plantarum in relation to the inoculation levels (ranging between 4 and $7 \log \mathrm{CFU} / \mathrm{cm}^{2}$ ) and the packaging materials are shown in Figure 1A. Its cell load decreased over time independently on the inoculation level and packaging materials. However, the decrease was significantly faster in cardboard compared to plastic materials. In fact, after $24 \mathrm{~h}$ of storage, the $L b$. plantarum cell load decreases were higher than $5 \log \mathrm{CFU} / \mathrm{cm}^{2}$ in cardboard and less than $3 \log \mathrm{CFU} / \mathrm{cm}^{2}$ in plastic material. In addition, on cardboard material, when the inoculation levels were of 4$5 \mathrm{log} \mathrm{CFU} / \mathrm{cm}^{2}, \mathrm{Lb}$. plantarum resulted under the detection limit $\left(0.5 \log \mathrm{CFU} / \mathrm{cm}^{2}\right)$ after 8 and $24 \mathrm{~h}$ of storing at environmental conditions $\left(25^{\circ} \mathrm{C}\right)$, respectively. By contrast, for plastic material, also considering the lowest inoculation level, the Lb. plantarum cell loads never decreased under the detection limits. 

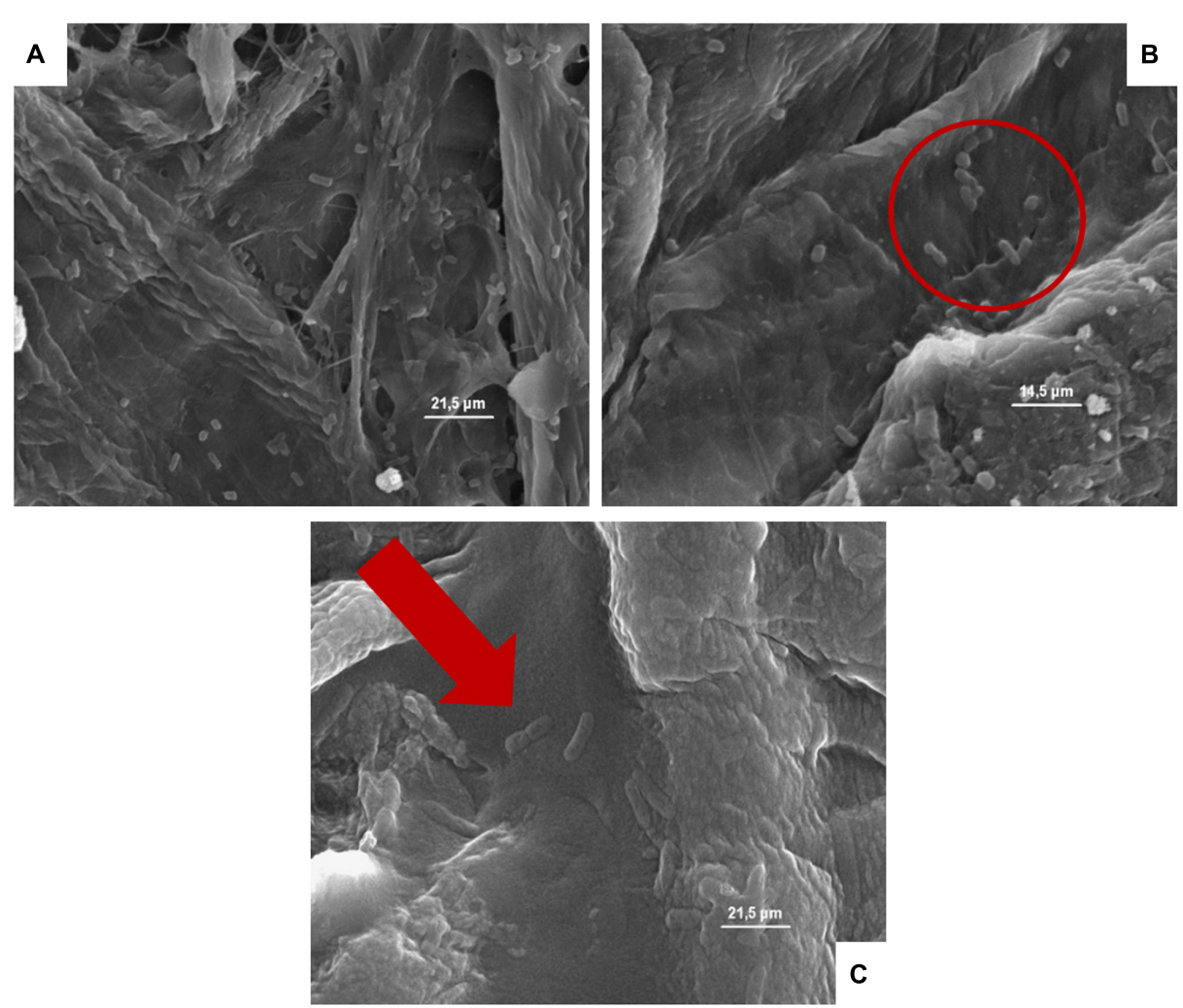

FIGURE 6 | (A-C) Presence of the inoculated Escherichia coli on cardboard surface after $1 \mathrm{~h}$ (A), $24 \mathrm{~h}$ (B), and $48 \mathrm{~h}$ (C). In $\mathbf{A}$ and $\mathbf{B}$ is evidenced the presence of pores and the entrapping of microbial cells between cardboard fibers. In $\mathbf{C}$, the $E$. coli lysis also of entrapped cells is outlined by the red harrow.

The results obtained for $P$. fluorescens, during the storage at environmental temperature, in relation to the cell loads inoculated (ranging between 3 and $6 \mathrm{log} \mathrm{CFU} / \mathrm{cm}^{2}$ ) and the packaging materials are shown in Figure 1B. Also for this spoilage bacteria, the same behavior of $L b$. plantarum was observed in relation to the packaging material. However, $P$. fluorescens resulted more sensitive to the environmental conditions, decreasing more quickly its viability both on plastic and cardboard materials. In fact, with an inoculation level of $3 \log \mathrm{CFU} / \mathrm{cm}^{2}$, it reached contamination levels under the detection limit after 1 and $8 \mathrm{~h}$ in cardboard and plastic, respectively. When the inoculation level increased to $4 \log \mathrm{CFU} / \mathrm{cm}^{2}, P$. fluorescens dropped under the detection limit after 1 and $48 \mathrm{~h}$ in cardboard and plastic materials, respectively.

Among the spoilage microorganisms considered, S. cerevisiae resulted the most resistant to the environmental conditions, showing the lowest cell load reductions (Figure 1C). In fact, its cell load decreases down to the detection limit were observed after $24 \mathrm{~h}$ only on cardboard surfaces and with a contamination level of about $3 \mathrm{log} \mathrm{CFU} / \mathrm{cm}^{2}$. However, significant differences in terms of $S$. cerevisiae cell loads between plastic and cardboard were observed independently on the inoculation levels and storage times. The reduction of the superficial cell load levels of all the spoilage microorganisms inoculated on the surfaces of the packaging materials clearly indicated the decrease of the contamination potential of cardboard compared to plastic material of packed fruit. The reduction of spoilage microorganism cross-contamination due to the packaging material is considered fundamental to increase the fruit shelf-life and quality. These results are in line with the outcomes obtained by Patrignani et al. (2016) that showed a reduction of potential cross-contamination by pathogens and spoiling microorganisms from packaging surfaces to fruits in cardboard compared to plastic.

\section{Cell Loads of Pathogenic Microorganisms in Relation to Inoculation Level, Packaging Material, and Storage Time}

The cell loads recorded during the storage at environmental temperature of $S$. enteritidis in relation to the inoculation levels (ranging between 2 and $5 \log \mathrm{CFU} / \mathrm{cm}^{2}$ ) and the packaging materials are shown in Figure 2A. The cell loads of this pathogenic species decreased over time independently on the inoculation level and packaging material used. However, the cell load decrease was significantly faster in cardboard compared to plastic materials.

In fact, with inoculation levels of about 2 and $3 \log \mathrm{CFU} / \mathrm{cm}^{2}$, $S$. enteritidis reached cell loads under the detection limit $\left(1 \log \mathrm{CFU} / \mathrm{cm}^{2}\right.$ ) after 1 and $8 \mathrm{~h}$ on cardboard and plastic surfaces, respectively. When the inoculation level increased to 


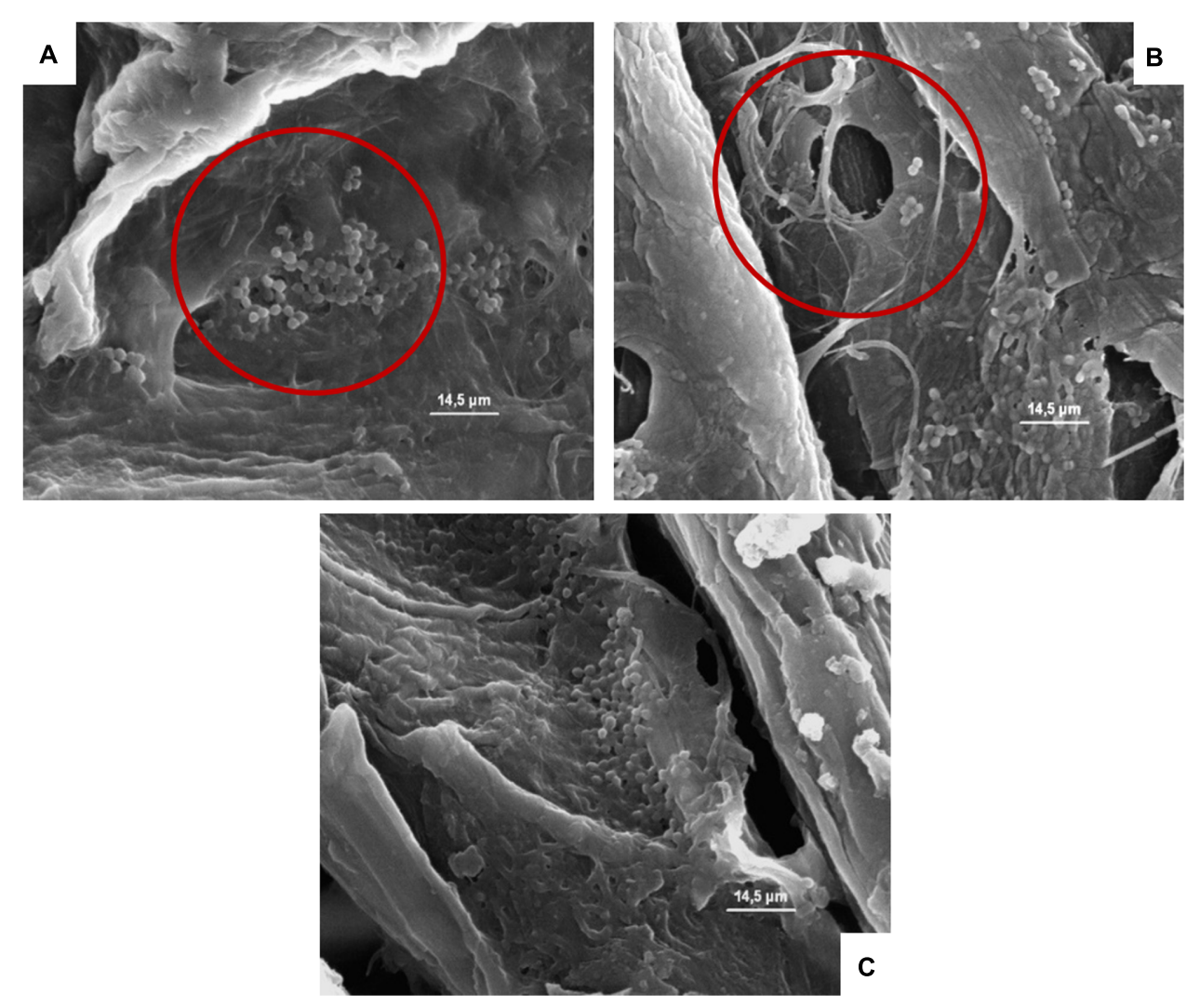

FIGURE 7 | (A-C) Presence of the inoculated L. monocytogenes on cardboard surface after $1 \mathrm{~h}$ (A), $24 \mathrm{~h}$ (B), and $48 \mathrm{~h}$ (C). In A-C the presence of pores and the entrapping of microbial cells between cardboard fibers are evidenced.

about $5 \log \mathrm{CFU} / \mathrm{cm}^{2}$, this microorganism decreased its cell loads under the detection limit after 8 and $48 \mathrm{~h}$ in cardboard and plastic surfaces, respectively. Similar results were obtained for E. coli, as shown in Figure 2B.

As shown in Figure 2C, with inoculation level of about $3 \log \mathrm{CFU} / \mathrm{cm}^{2}$, also L. monocytogenes, a dangerous psychotropic Gram-positive food pathogen, decreased its cell load under the detection limit within 1 and $8 \mathrm{~h}$ of storage at environmental conditions on cardboard and plastic surfaces, respectively. As expected, this pathogenic species was more resistant to the environmental conditions compared to the Gram-negative species considered (S. enteritidis, E. coli, and P. fluorescens). In any case, the decrease of $L$. monocytogenes cell load was significantly higher on cardboard that on plastic surface. On the other hand, it is widely reported that Gram-positive bacteria and yeasts could survive longer at environmental conditions on inert surfaces (Robine et al., 2000; Tang, 2009).

The reduction of the superficial cell load levels of all the pathogenic microorganisms inoculated on the surfaces of the packaging materials clearly showed the reduction of the contamination risk in fruit packaged in cardboard compared to that packaged in plastic material. The reduction of pathogen cross contamination due to the packaging material is fundamental to increase the fruit safety features and to decrease the occurrence of food borne diseases associated to the intake of fruits and vegetables. The role contact surface in the contamination of food is widely recognized and consequently in Europe ECR 8522004 regulates and specify the measures that have be carried out to guarantee a safe contact between products and packaging material and to avoid chemical and microbial contamination. For example, there are stringent limits for pathogens in the Annex $1^{1}$ (Carpentier and Barre, 2012).

\section{Scanning Electron Microscope Analyses}

To understand if the cell load reductions observed were due to the cell viability losses or to the entrapment of the microorganisms within the cardboard fibers, the inoculated surfaces were studied through SEM technique.

The microphotographs obtained showed, independently on the microbial species considered, the great capability of cardboard to entrap the microbial cells contributing in a significant manner to reduce the superficial contamination level in terms of both spoilage and pathogenic microorganisms and, consequently, its role in the contamination of packed fruit with the same microorganisms. In fact, the microstructure of the cardboard showed pores ranging between few to hundreds of

\footnotetext{
${ }^{1}$ Recommendations for an environmental monitoring program for Listeria monocytogenes in processing areas. Guidelines on the Application of General Principles of Food.
} 

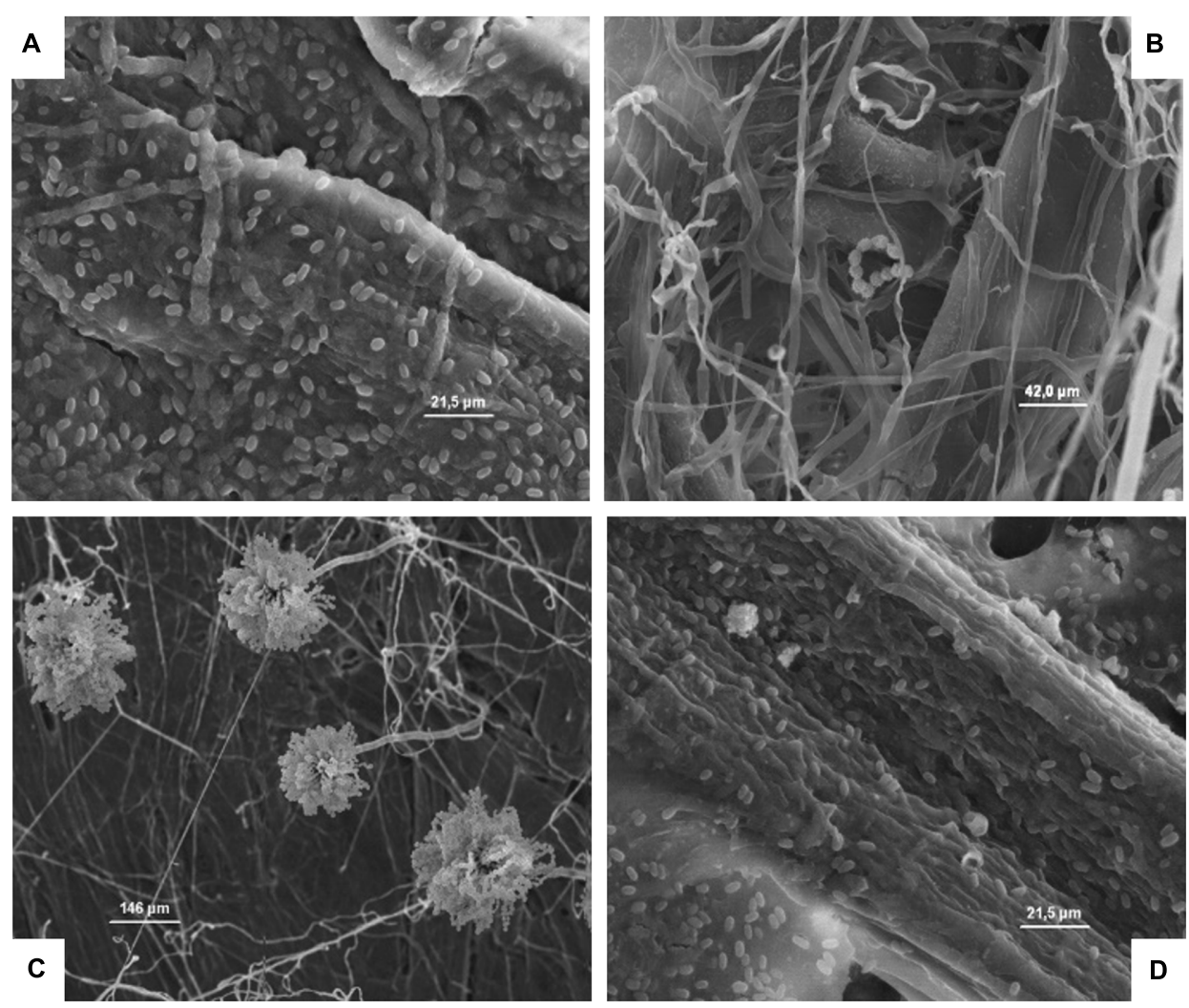

FIGURE 8 | (A-D) Presence of the inoculated A. flavus on cardboard surface after 1 (A), 24 (B), and $48 \mathrm{~h}$ (C) in the presence of high relative humidity, and after $48 \mathrm{~h}$ in a dry environment (D). The spores resulted not germinated after $1 \mathrm{~h}$ (A), while after 24 and $48 \mathrm{~h}$ in the presence of high relative humidity a germination of the spore is clear and evidenced by the presence of mycelia and conidiophore (B,C). By contrast, after $48 \mathrm{~h}$ in a dry environment no mycelia growth was observed.

microns (Figure 3A). Microbial cells considered in these studies have dimensions ranging between 0.7 (L. monocytogenes) to about $10 \mu \mathrm{m}$ (S. cerevisiae).

By contrast, plastic material (Figure 3B) showed a smooth, plane, and homogeneous surface without hole, and micro-pores able to entrap microorganisms.

On the other hand, it is well known that the microbial contamination of surfaces, the survival of microorganisms and their eventual growth or death rates depend on their features and conditions. For example, characteristics as roughness and porousness or the execution of a sanitization process strongly affect microbial survival (Ismail et al., 2013; Montibus et al., 2016). By contrast, on the plastic surfaces (even if new and sanitized very well) were evident cuts and scratches due to the re-usage able to recover in case of inappropriate cleaning and sanitization processes organic matter in which microorganisms are reported to proliferate.

The role of microstructure of packaging materials in the transferring of microorganism to fruits had been recently underlined also by Montibus et al. (2016). These authors showed through a challenge test that poplar crates, being a porous material, guaranteed a reduced cross-contamination Penicillium expansum conidia and E. coli of packaged apples. These authors showed that $P$. expansum conidia survived but did not grow on wood specimens if the absorption of humidity was prevented. They showed also that E. coli decreased its cell loads on the poplar crate surfaces after $1 \mathrm{~h}$ from the inoculation. Also, these authors attributed the reduction of superficial cell loads and of the apple contamination potential to the entrapping capability of poplar wood without demonstrating it with SEM analyses. In fact, wood, analogously to corrugated surface is characterized by a high roughness and porousness. For example, poplar has several pores of small size and regularly placed throughout a growth ring (Jacquiot et al., 1973).

Figures 4-8, relative to Lb. plantarum, S. cerevisiae, E. coli, L. monocytogenes vegetative cells, and A. flavus conidia after 1 , 24 , and $48 \mathrm{~h}$, show the microorganisms entrapped within the cardboard fibers and pores. After $24-48 \mathrm{~h}$, depending on the microbial species considered, the lyses also of the entrapped cells are evident. This means that also the entrapped cells, due to the lack of nutrient inside the cardboard fibers, die over time. This phenomenon is particularly evident in S. cerevisiae and L. monocytogenes. Also, the conidia of A. flavus were entrapped within the cardboard fibers. No mycelium growth was observed when the cardboard was completely dried after inoculation while with high relative humidity the growth of the mold mycelia was evident inside cardboard after 24 and $48 \mathrm{~h}$. Since molds are important spoilage agents of fruits and vegetables, 
their proliferation on packaging material should be avoided in order to prevent the cross-contamination of the packaged fruits. Consequently, the storage of cardboard packaging materials at low relative humidity is fundamental to prevent the mold growth and the increase of their cell loads on surfaces in contact with fresh produces (Hladikova et al., 2015; Patrignani et al., 2016). The increase of the number of the cells entrapped within the corrugated fibers clearly demonstrates the reduction of the superficial contamination level and, consequently, the microbial capability to cross-contaminate the packed material.

Due to the compact structure (lacking pores able to entrap microorganisms) of plastic, the SEM analyses for this material were performed inoculating only $L b$. plantarum. The results showed that the cells of this microorganism remained on the surface acting a major role in the cross-contamination of the packed products in accordance with the cell load data.

\section{CONCLUSION}

The data clearly demonstrated that cardboard materials, if correctly stored, reduce the potential of packaging to the cross-contamination of food due to a quicker viability loss by spoilage and pathogenic microorganisms compared to the

\section{REFERENCES}

Alegre, I., Abadias, M., Anguera, M., Oliveira, M., and Vinas, I. (2010). Factors affecting growth of foodborne pathogens on minimally processed apples. Food Microbiol. 27, 70-76. doi: 10.1016/j.fm.2009.08.005

Bae, Y. M., Baek, S. Y., and Lee, S. Y. (2012). Resistance of pathogenic bacteria on the surface of stainless steel depending on attachment form and efficacy of chemical sanitizers. Int. J. Food Microbiol. 153, 465-473. doi: 10.1016/j. ijfoodmicro.2011.12.017

Barnes, L. M., Lo, M. F., Adams, M. R., and Chamberlain, A. H. L. (1999). Effect of milk proteins on adhesion of bacteria to stainless steel surfaces. Appl. Environ. Microbiol. 65, 4543-4548.

Binderup, M., Pedersen, G. A., Vinggaard, A. M., Rasmussen, H., Rosenquist, H., and Cederberg, T. (2002). Toxicity testing and chemical analyses of recycled fibre-based paper for food contact. Food Addit. Contam. 19, 13-28. doi: 10.1080/ 02652030110089878

Brandwein, M., Al-Quntar, A., Goldberg, H., Mosheyev, G., Goffer, M., MarinIniesta, F., et al. (2016). Mitigation of biofilm formation on corrugated cardboard fresh produce packaging surfaces using a novel thiazolidinedione derivative integrated in acrylic emulsion polymers. Front. Microbiol. 7:159. doi: 10.3389/fmicb.2016.00159

Callejón, R. M., Rodríguez-Naranjo, M. I., Ubeda, C., Hornedo-Ortega, R., Garcia-Parrilla, M. C., and Troncoso, A. M. (2015). Reported foodborne outbreaks due to fresh produce in the United States and European Union: trends and causes. Foodborne Pathog. Dis. 12, 32-38. doi: 10.1089/fpd.20 14.1821

Campos, D., Piccirillo, C., Pullar, R. C., Castro, P. M., and Pintado, M. M. (2014). Characterization and antimicrobial properties of food packaging methylcellulose films containing stem extract of Ginja cherry. J. Sci. Food Agric. 94, 2097-2103. doi: 10.1002/jsfa.6530

Carpentier, B., and Barre, L. (2012). Guidelines on Sampling the Food Processing Area and Equipment for the Detection of Listeria monocytogenes. Available at: https://ec.europa.eu/food/sites/food/files/safety/docs/biosafety_ fh_mc_guidelines_on_sampling.pdf [accessed June 26, 2017].

De Candia, S., Morea, M., and Baruzzi, F. (2015). Eradication of high viable loads of Listeria monocytogenes contaminating food-contact surfaces. Front. Microbiol. 6:733. doi: $10.3389 /$ fmicb. 2015.00733 plastic ones. This phenomenon was observed for all the target microorganisms considered, except for molds which were able to proliferate when the absorption of water was not prevented during the storage. The SEM microphotographs showed the reduction of superficial contamination on cardboard surfaces was due to the entrapping of the microbial cells within the fibers and the pores of this packaging material and their death overtime due to the absence of water and nutrients. These results represent an objective evidence of previous findings on the reduced cross-contamination potential of corrugated compared with plastic packaging materials used in fruit and vegetable sector. Moreover, these results outlined the importance of storage and supply chain conditions (hygiene and low humidity) to prevent the mold growth and the increase of their role in the cross-contamination of packed foods.

\section{AUTHOR CONTRIBUTIONS}

LS, DS, and MB contributed to the acquisition, analysis, and interpretation of the data of the work. FP, CC, LG, and RL contributed to the design of the work, and the drafting and revision of the manuscript.

Edelmann, R. E., and Klomparens, K. L. (1994). The ultrastructural development of sporangiospores in multispored sporangia of Zygorhynchus heterogamus with a hypothesis for sporangial wall dissolution. Mycologia 86, 57-71.

Francis, G. A., Gallone, A., Nychas, G. J., Sofos, J. N., Colelli, G., Amodio, M. L., et al. (2012). Factors affecting quality and safety of fresh-cut produce. Crit. Rev. Food Sci. Nutr. 52, 595-610. doi: 10.1080/10408398.2010. 503685

Heaton, J. C., and Jones, K. (2008). Microbial contamination of fruit and vegetables and the behaviour of enteropathogens in the phyllosphere: a review. J. Appl. Microbiol. 104, 613-626. doi: 10.1111/j.1365-2672.2007. 03587.x

Hladikova, Z., Kejlova, K., Sosnovcova, J., Jirova, D., Vavrous, A., Janousek, S., et al. (2015). Microbial contamination of paper-based food contact materials with different contents of recycled fiber. Czech J. Food Sci. 33, 308-312. doi: 10.17221/645/2014-CJFS

Ismaïl, R., Aviat, F., Michel, V., Le Bayon, I., Gay-Perret, P., Kutnik, M., et al. (2013). Methods for recovering microorganisms from solid surfaces used in the food industry: a review of the literature. Int. J. Environ. Res. Public Health 10, 6169-6183. doi: 10.3390/ijerph10116169

Jacquiot, C., Trenard, Y., and Dirol, D. (1973). Atlas d'anatomie des bois des angiospermes (essences feuillues), Vol. 2. Paris: Centre Technique du Bois.

Kusumaningrum, H. D., Riboldi, G., Hazeleger, W. C., and Beumer, R. R. (2003). Survival of foodborne pathogens on stainless steel surfaces and crosscontamination to foods. Int. J. Food Microbiol. 85, 227-236. doi: 10.1016/S01681605(02)00540-548

Levi, M., Cortesi, S., Vezzoli, C., and Salvia, G. (2011). A comparative life cycle assessment of disposable and reusable packaging for the distribution of Italian fruit and vegetables. Packag. Technol. Sci. 24, 387-400. doi: 10.1002/pts.946

Mafu, A. A., Plumety, C., Deschenes, L., and Goulet, J. (2011). Adhesion of pathogenic bacteria to food contact surfaces: influence of $\mathrm{pH}$ of culture. Int. J. Microbiol. 2011:972494. doi: 10.1155/2011/972494

Montibus, M., Ismail, R., Michel, V., Federighi, M., Aviat, F., and Le Bayon, I. (2016). Assessment of Penicillium expansum and Escherichia coli transfer from poplar crates to apples. Food Control 60, 95-102. doi: 10.1016/j.foodcont.2015. 07.025

Oliveira, M., Viñas, I., Anguera, M., and Abadias, M. (2012). Fate of Listeria monocytogenes and Escherichia coli $\mathrm{O} 157: \mathrm{H} 7$ in the presence of natural 
background microbiota on conventional and organic lettuce. Food Control 25, 678-683. doi: 10.1016/j.foodcont.2011.12.002

Patrignani, F., Siroli, L., Gardini, F., and Lanciotti, R. (2016). Contribution of two different packaging material to microbial contamination of peaches: implications in their microbiological quality. Front. Microbiol. 7:938. doi: 10. 3389/fmicb.2016.00938

Robine, E., Dérangère, D., and Robin, D. (2000). Survival of a Pseudomonas fluorescens and Enterococcus faecalis aerosol on inert surfaces. Int. J. Food Microbiol. 55, 229-234. doi: 10.1016/S0168-1605(00)00188-184

Scallan, E., Hoekstra, R. M., Angulo, F. J., Tauxe, R. V., Widdowson, M. A., Roy, S. L., et al. (2011). Foodborne illness acquired in the United States-major pathogens. Emerg. Infect. Dis. 17, 7-15. doi: 10.3201/eid1701.P11101

Shi, X., and Zhu, X. (2009). Biofilm formation and food safety in food industries. Trends Food Sci. Technol. 20, 407-413. doi: 10.1016/j.tifs.2009.01.054

Siroli, L., Patrignani, F., Serrazanetti, D. I., Tabanelli, G., Montanari, C., Tappi, S., Rocculi, P., et al. (2014). Efficacy of natural antimicrobials to prolong the shelflife of minimally processed apples packaged in modified atmosphere. Food Control 46, 403-411. doi: 10.1016/j.foodcont.2014.05.049

Suominen, I., Suihko, M. L., and Salkinoja-Salonen, M. (1997). Microscopic study of migration of microbes in food-packaging paper and board. J. Ind. Microbiol. Biotechnol. 19, 104-113. doi: 10.1038/sj.jim.2900424

Sowden, L. C., and Walker, T. (1988). A procedure for the study by scanning electron microscopy of flocculating cells of the fission yeast Schizosaccharomyces pombe. Can. J. Microbiol. 34, 577-582. doi: 10.1139/ m88-096

Tang, J. W. (2009). The effect of environmental parameters on the survival of airborne infectious agents. J. R. Soc. Interface 6, 737-746. doi: 10.1098/rsif.20 09.0227
Turtoi, M., and Nicolau, A. (2007). Intense light pulse treatment as alternative method for mould spores destruction on paper-polyethylene packaging material. J. Food Eng. 83, 47-53. doi: 10.1016/j.jfoodeng.2006. 11.017

Uhlich, G. A., Cooke, P. H., and Solomon, E. B. (2006). Analyses of the red-dryrough phenotype of an Escherichia coli O157:H7 strain and its role in biofilm formation and resistance to antibacterial agents. Appl. Environ. Microbiol. 72, 2564-2572. doi: 10.1128/AEM.72.4.2564-2572.2006

Valeriano, C., de Oliveira, T. L. C., de Carvalho, S. M., das Graças Cardoso, M., Alves, E., et al. (2012). The sanitizing action of essential oil-based solutions against Salmonella enterica serotype Enteritidis S64 biofilm formation on AISI 304 stainless steel. Food Control 25, 673-677. doi: 10.1016/j.foodcont.2011. 12.015

Wilks, S. A., Michels, H. T., and Keevil, C. W. (2006). Survival of Listeria monocytogenes scott a on metal surfaces: implications for cross-contamination. Int. J. Food Microbiol. 111, 93-98. doi: 10.1016/j.ijfoodmicro.2006.04.037

Conflict of Interest Statement: The authors declare that the research was conducted in the absence of any commercial or financial relationships that could be construed as a potential conflict of interest.

Copyright (c) 2017 Siroli, Patrignani, Serrazanetti, Chiavari, Benevelli, Grazia and Lanciotti. This is an open-access article distributed under the terms of the Creative Commons Attribution License (CC BY). The use, distribution or reproduction in other forums is permitted, provided the original author(s) or licensor are credited and that the original publication in this journal is cited, in accordance with accepted academic practice. No use, distribution or reproduction is permitted which does not comply with these terms. 